\title{
A Critical Exploration of How Language-of-Instruction Choices Affect Educational Equity
}

\author{
Lizzi O. Milligan, Zubeida Desai and Carol Benson
}

In many parts of the world, children are forced to sink or swim in educational systems that rely on a single dominant language as the medium of instruction. Target 4.1 of the Sustainable Development Goals (SDGs) calls for all 193 signatory countries to 'ensure that all girls and boys complete free, equitable and quality primary and secondary education leading to relevant and effective learning outcomes' by 2030, and Target 4.5 extends that mandate to 'eliminate gender disparities in education and ensure equal access to all levels of education ... for the vulnerable, including persons with disabilities, indigenous peoples and children in vulnerable situations' (UNGA, 2015b). As professionals who have spent our careers promoting quality education, we find it unacceptable that there is no specific mention of language in those targets and its essential role in providing access to literacy and quality education. In this chapter, we draw on relevant literature and case studies from Cambodia, South Africa, and Rwanda to show that Targets 4.1 and 4.5 will not be met unless more emphasis is placed on the language(s) through which learners are taught. We will argue that language(s) of instruction are either central enablers or barriers to educational equity, since it is through these languages that a learner must gain access to initial and continuing literacy as well as learn to think critically and interact with teachers and peers around curricular content.

In policy discussions about language-in-education, and about education more broadly, there is often a tendency among those promoting equity to focus on institutional access. In his seminal paper on 'epistemological access', Morrow (1993) argues that there is no guarantee that epistemological access, that is, meaningful access to the knowledge, skills, and values that are the content of the curriculum, will take place in contexts where institutional access has been broadened to be more inclusive. We define educational equity as the normative process that enables equality of educational opportunities for such epistemological access. Implicit to this is an understanding that to achieve this

(C) KONINKLIJKE BRILL NV, LEIDEN, 2020 | DOI: 10.1163/9789004430365_005 
in practice there is no 'one size fits all' solution; rather this will entail the use of different approaches to give learners such opportunities. Equity is thus a full realisation of rights, beyond mere access to education, to include the ways that learners experience education and the outcomes that they are able to achieve.

For educational equity to be addressed, as Benson (2016) has pointed out, at least three questions must be answered about language use in the classroom: First, is the learner taught and assessed in a language $\mathrm{s} / \mathrm{he}$ understands and speaks well? Second, does instruction draw on the learner's prior experiences and resources to construct new knowledge? And third, are teachers proficient in the language(s) of instruction? These questions will guide our critical exploration of language-of-instruction choices and educational equity.

Globally, there are millions of children who learn in a dominant language. For many, this is a language foreign to entire populations, as is the case of Portuguese for most Mozambicans, French for most Malians, or Standard Arabic for most Moroccans. In these and other countries, there are significant portions of national populations that lack access to the dominant language, e.g., indigenous people in Cambodia for whom Khmer is a new language. The issue often goes deeper than language to include undervaluing and outright discrimination against learners whose ethnic, cultural, or economic backgrounds differ from the dominant 'norm'. In recent years, the use of English as a medium of instruction (EMI) has grown significantly in many parts of the world, driven by an assumed relationship between proficiency in a 'global' language and economic development (Casale \& Posel, 2011; Dearden, 2014). Comparable drives for other dominant languages continue in parallel, revealing a second assumed relationship - that between using a language as medium of instruction and actually learning that language. This latter assumption is difficult to prove true given the large repetition, failure, and drop-out rates in systems using a foreign language of instruction, as will be discussed later. The problem is grave. A recent policy paper written for the British Council reinforces an evidencebased principle, adopted by major donors and education partners in Juba in 2012, stating that 'introducing EMI at primary level in low- or middle-income countries [for whom English is not a mother tongue] is not a policy decision or practice that should be supported' (Simpson, 2017, p. 11). The same could and should be said for Portuguese, French, and other exogenous languages still widely used in education in postcolonial countries, as well as dominant languages that are foreign to large groups of learners. 
Decades of research demonstrate that the use of a dominant language as the medium of instruction negatively affects learners' ability to both learn that language and access the wider curriculum (e.g., Mohanty, 2009; Nomlomo, 2009; Brock-Utne, 2010; Heugh, 2009; Desai, 2013). The results are strikingly similar: limited access to schooling; high repetition, failure, and dropout rates; poor quality of education; and low learner self-esteem - all of which are well documented (e.g., Ball, 2010). While acknowledging that the language factor does not stand alone, Walter (2008) has found a distributional relationship between learners' access to education in their first language (L1) and level of national development, demonstrating that countries that do not provide access to L1 education experience the lowest levels of literacy and educational attainment worldwide. This evidence runs concurrent with recognition of a so-called 'learning crisis' (UNESCO, 2014f), with figures from 2014 suggesting that at least 250 million children do not have access to basic numeracy and literacy skills in any language, even by the end of grade 4 (UNESCO, 2014f). For example, the most recent Progress in International Reading Literacy Study (PIRLS) results indicate that around $78 \%$ of grade 4 learners in South Africa do not have basic reading skills by the end of the school year (Howie et al., 2017, p. 11).

Thus far we have tended to focus on the role of language in facilitating or obstructing learning. But language is also a marker of identity and as such can play an important role in influencing one's self-esteem and confidence, depending on the language(s) with which one identifies. There are resources that teachers can use in the classroom to help learners and students make the connection between their language, their identity, and their learning, and to see their language as a communicative resource. In an interesting chapter on using a multiliteracies pedagogical approach to developing texts, Cummins (2006) explores the use of multimedia to help pupils in a school in Canada to develop what he refers to as 'identity texts'. By encouraging learners to invest in their identities, they are able to develop bilingual resources. It is important that young people are proud of their identities so that learning moves seamlessly from the familiar to the less familiar. ${ }^{1}$

Underpinning policy assumptions about the merits of EMI is a polarisation of debates between equity and efficiency. Equity (as well as equality) has often been equated with redress and human rights, while efficiency signals economic development. The argument made is that the use of local languages 'ghettoises' learners and prevents them from being successful in an increasingly global world. The solution posited is the use of a dominant language as the medium of instruction because it will open doors for learners. Post-Apartheid South Africa is a clear example of this approach (Desai, 20oo; South Africa, NECC, 
1993). This line of thinking can be robustly challenged since using a dominant language as the medium of instruction has been shown in that case to be a clear obstacle to equity.

Learning in a dominant language affects the educational opportunities available to learners, particularly learners from disadvantaged backgrounds. Numerous studies have shown the negative impact on girls (Hovens, 2002; Benson, 2005) and children from lower socioeconomic groups (Fleisch, 2008; Smith, 2011), poor urban areas and remote rural areas (Benson \& Wong, 2017; Evans \& Cleghorn, 2012; Milligan, Clegg, \& Tikly, 2016), nondominant groups (Benson \& Wong, 2017), and conflict-affected areas (Dryden-Peterson, 2015). It is important to note that with increased globalisation and the movement of people, the mismatch between learners' own languages and the medium of instruction is by no means just a 'developing country problem'. Even in highincome countries, the use of a single language of instruction is problematic because it exacerbates differences between people from dominant and nondominant social groups. However, evidence suggests that it is particularly problematic in low-income and postcolonial contexts where there is greater fracture between local and official (and usually ex-colonial) languages (Milligan \& Tikly, 2016), and where the negative effects are compounded by health and safety issues, particularly for girls, as well as low levels of teacher education, content-heavy and inappropriate curricula, and lack of adequate school facilities (Benson, 2016).

Given this scenario of low educational outcomes among learners taught through an unfamiliar language, why is mother tongue-based multilingual education (or MLE) treated like such a 'hot potato' by national policymakers? The answer lies in the fact that medium-of-instruction issues are seldom resolved on pedagogical grounds, but rather on political, economic, or cultural ones. In the NEPI Report ${ }^{2}$ on language in South Africa, the authors describe what they call 'common-sense assumptions' (South Africa, NECC, 1992). Politically, the assumption would be that a multilingual country needs a single unifying language; economically, it would be that using multiple languages is too costly; and culturally, it would be that using diverse languages could cause ethnic rivalry. All of these could be seen as representing a monolingual habitus (Gogolin, 2009), also known as a monoglossic ideology (García, 2009), meaning that people assume it is necessary to choose one single language, even though it is contrary to the nature of multilingual societies.

Each of these assumptions has been effectively challenged. On the political front, one can argue, as Bamgbose (1991) did long ago, that in a multilingual context, those citizens proficient in two (or more) languages are more integrated than those proficient in a single language, even if that language happens 
to be the dominant or official language. Indeed, recent research shows that jobs increasingly call for multiple language skills (Duchêne and Heller, 2012). It has been claimed that multilingualism is the true lingua franca of a globalised world (Prah \& Brock-Utne, 2009; see also Benson \& Elorza, 2015). The arguments on the economic front assume that a choice must be made between acquiring either a local language or a language of national or international communication. There is no disputing that in a country like South Africa, there is a need for a language like English for people to be able to interact with wider levels of society, particularly the outside world. But it is problematic to make the possibility of such interaction the basis of language-in-education policies for speakers of African languages who might never have the opportunity to go beyond their local village or township if they are denied meaningful access to education. As far as cultural arguments go, it is not only language that divides people. There are many other factors such as class inequalities, distribution of resources, and power relations that lead to rivalry between groups in a country. May takes up this point in the context of New Zealand when he defends Maori-medium education against the accusation that it is separatist and a 'retrenchment in the past' (May, 2004, p. 34). In his view, Maori-medium education is simply making available to Maori children choices that are taken for granted by majority language speakers. Despite these persuasive arguments, we continue to see dominant languages used as media of instruction in the majority of countries in the Global South, especially in the later years of primary and secondary schooling.

\section{The Role of Language(s) of Instruction in SDG 4}

Considering the significant evidence base presented in the previous section, it may be surprising that a review of key policy documents related to SDG 4 reveals that there is very limited attention paid to the language of instruction. In a key UNESCO publication about the SDGs entitled Sustainable Development Begins with Education, there is only one mention of language in a section entitled 'Education Is Essential for the Justice System to Function', which discusses how, in the case of Sierra Leone, 'many people with little education cannot use the formal court system because it operates in English ... [and] some people only speak local languages' (UNESCO, 2014e, p. 14). This is all the more disturbing because Sierra Leonean adults are typically multilingual in indigenous languages and Krio, a widely spoken lingua franca (Simons \& Fennig, 2017). In the Incheon Declaration and Education 203o Framework for Action (WEF, 2015), we see slightly more attention paid to language: it is mentioned a total of 12 times. While it is positive to see language primarily discussed in this document 
in relation to learners' home languages, the discussion of language is too narrowly focused on both the early years and institutional access. Language is only mentioned once in relation to learning:

Particular attention should be paid to the role of learners' first language in becoming literate and in learning. Literacy programmes and methodologies should respond to the needs and contexts of learners, including through the provision of context-related bilingual and intercultural literacy programmes. (WEF, 2015, p. 20)

As noted in the previous section, the language issue is often seen as a deeply political and potentially contentious issue and one that often is determined primarily away from the sphere of education. This political dimension may, at least partially, explain the hesitancy with which language-related recommendations are made, as is evident in comments such as the following: Teaching and learning in the home language 'should be encouraged ... where possible' and while 'taking into account different national and subnational realities' (WEF, 2015, p. 13). No mention is made of the language of instruction for secondary education, even though Target 4.1 obliges countries to treat primary and secondary education as equally important in terms of free and compulsory access.

The failure to mention language has been addressed by professionals in the field, such as Benson (2016) and Kosonen (2017), whose background papers for the Unesco Global Education Monitoring Reports point out language-related omissions and suggest how relevant language-related data should be collected and used to monitor educational quality. Thus far they remain suggestions, hidden in a single optional indicator in a UIS metadata document (UIS, 2018b). This single indicator (4.5.2; see Appendix 2) refers to 'the percentage of students in primary education whose first or home language is the language of instruction'. Despite the much greater emphasis on learning outcomes and a wider conceptualisation of basic education in SDG 4 more broadly, here we see language relegated to a single indicator narrowly focused on primary education and its availability, despite the intense efforts of many professional educators' negotiations.

Language is, thus, not currently recognised as an equity issue. Firstly, the focus on availability of a home language as language of instruction suggests simply equal provision. This reflects similar concerns voiced by Milligan (2014), among others, about the misguided use of gender parity measures as indicative of gender equity in the MDG era. In the same way that gender parity only reflects quantitative measures and does not address the root sociocultural 
gendered norms, a focus on home language availability does not allow for a more complex understanding of the ways that language of instruction can enable or disable equal opportunities to learn and so an equitable educational system. Secondly, a focus on the proportion of learners learning in a home language leads us to consider the groups of children who may not be included in that proportion. This may mean that the most marginalised become more marginalised, particularly those who do not speak the majority home language, such as refugees in an urban setting. Finally, it seems that, as with national policies, language is not seen as a key pedagogical issue. Overall, there is further need for learners to see themselves reflected in the curriculum as well as teaching and learning materials. The following sections argue for the importance of seeing language as a central factor in inclusive and equitable learning.

\section{$4 \quad$ Lessons for Policy and Practice}

Through exploration of recent research in South Africa, Rwanda, and Cambodia, this section of the chapter focuses on the key challenges to the achievement of SDG 4 and lessons that can be learned that will facilitate more equitable learning throughout the basic education cycle. Given the highly political nature of the language-of-instruction debate, we focus on both policy and practice. One of Desai's (2000) arguments countering objections to mother tongue-based multilingual education is that languages develop through use, and the more we use local languages, the more they will develop, thereby facilitating learning for young children. It is in this spirit that we proceed with our arguments for a multilingual approach to education and follow the argument put forward by the Asia-Pacific Multilingual Education Working group:

We live in a multilingual world. ... Yet, most education systems ignore this multilingual reality. Equitable quality education and lifelong learning for all is only possible where education responds to and reflects the multilingual nature of the society. (Wisbey, 2016, p. 2)

In the three multilingual cases described here, there is some recognition of the potential for MLE to address equity issues. Each case provides a particular set of lessons - both positive and negative - with implications for other contexts.

\subsection{South Africa}

In terms of policy, South Africa would appear to be well ahead of other countries in recognising its multilingual realities. South Africa's language policy of 
11 official languages (RSA, 1996) has been internationally acclaimed as progressive, liberal, and unique (see Adams, 1999; Deumert, Inder, \& Maitra, 2005). Yet, a closer examination of language policy in practice reveals that African languages continue to be used in limited domains (Deumert et al., 2005; Mazrui \& Mazrui, 1998). Nowhere is this more apparent than in education. The South African Department of Education's Language in Education Policy Document of July 1997 proclaims that schools, through their governing bodies, can choose any of the official languages as the language of learning and teaching, within the bounds of practicability. Despite such a wide choice, the reality is that most schools in African townships continue to use English as the sole medium of instruction after grade 3, particularly with regard to assessment practices (Desai, 2013; Murray, 2002; Vinjevold, 1999; Ziegler, 2013).

Despite the July 1997 policy document (RSA, DoE, 1997) ${ }^{3}$ providing a fairly progressive framework for developing language in education policy, supposedly allowing parents (and learners) the right to choose the medium of instruction, the majority of African primary school learners find themselves at a disadvantage. Since there is no infrastructure in terms of teacher training or materials development to back parental decisions, choice becomes rather meaningless in practice. The picture that continues to emerge from schools is bleak - most learners have difficulty coping with the demands of using English as a medium in primary schools (Desai, 1999; Langenhoven, 2010; Nomlomo, 2008, 2009). Such difficulties are usually carried into high school and tertiary institutions. Even those learners who display a level of proficiency in English at what Cummins (1980, p. 175) calls the 'basic interpersonal communication skills' level, have difficulty with reading and writing tasks. Generally, the learning experience is a frustrating one for both learners and teachers. Despite this clear disjuncture between policy and practice, government officials can be overly eager to see quick results and not prepared to invest in long-term plans to address the many aspects of extending mother tongue instruction beyond the initial years. The only curriculum change has been that English is now introduced as a subject in grade 1 at schools catering to speakers of African languages (RSA, DBE, 2010a).

In light of these pedagogical concerns, the Language of Instruction in Tanzania and South Africa (LOITASA) Project - a longitudinal study spreading over three years (grades 4-6) and involving two primary schools in urban townships in the Western Cape - was undertaken. At each school, one class constituted the experimental group and the other a control group. The experimental group was taught science and geography (mathematics replaced geography in the second phase) in Xhosa while the control group was taught these subjects in English (see Brock-Utne, Desai, and Qorro, 2003, 2004, 2005, 2006; Brock-Utne, Desai, Qorro, \& Pitman, 2010; Desai, Qorro, \& Brock-Utne, 2010, 2013; Qorro, 
Desai, \& Brock-Utne, 2008, 2012, for more details on the research design and findings of the LOITASA Project). The project aimed to find out whether this switch to the mother tongue would be in the best interests of the learners for the acquisition of knowledge in science and geography (later mathematics) and development of English acquisition. Nomlomo (2008, p. 88) outlines the findings in relation to science learning as follows:

Learners developed high self-esteem and better confidence as they participated in classroom activities in their own language. They were spontaneous in responding to teachers' questions and they could express themselves clearly in their mother tongue (isiXhosa). Their written work made more sense than their counterparts who were taught through the medium of English. They could elaborate on issues, making use of complex sentences, which showed originality and better understanding of Science concepts.

The positive impact was also seen in learning outcomes, with learners in the isiXhosa-medium class consistently outperforming their counterparts who were taught in English. For example, the pass rate in science in grade 5 for the isiXhosa class ranged from 70 to $86 \%$ (Nomlomo, 2009). This supports the work of Langenhoven, also in South Africa, who has shown that 'when pupils use their mother tongue to read and talk about a topic, they construct meaning, making sense of their world and thus generating a better understanding of scientific concepts instead of memorizing scientific facts' (Langenhoven, 2010, p. 135).

Despite these important gains made by the project, as captured in the LOITASA edited volumes (Brock-Utne et al., 2003, 2010), Desai has also reflected on the wider challenges that are presented by the South African educational context, showing that learning in both the control and experimental groups was characterised by a teacher-centred approach, with children spending most of their time listening or producing choral responses:

Mother tongue education is a necessary but not a sufficient condition to remedy such teacher-centred approaches in the classroom, particularly in subjects such as Natural Science where it is expected that pupils' natural curiosity and higher-order thinking are to be encouraged. An intimacy with subject knowledge on the part of the teachers is needed to develop a confidence and boldness in teachers which, in turn, could lead to greater pupil involvement in learning. (Desai, 2010, p. 210) 
This is a point also taken up by Cummins (2009, p. 20) when he states that 'bilingual education ... is not by itself a panacea for underachievement'. This does not mean that mother tongue-based education should not be promoted. One needs to bear in mind that the one variable that remains constant in educational research is the correlation between socioeconomic status (SES) and academic achievement. This point has recently been corroborated by the PIRLS 2016 Report on International Results in Reading. It states that, on average, students' higher reading achievement was linked to their attending schools:

- With more affluent than economically disadvantaged students

- Where a higher proportion of their peers had early reading and writing skills when entering first grade

- Where instruction was not affected by reading resource shortages. (Mullis et al., 2017, p. x)

According to PIRLS analyses, language of instruction remains an important factor and clearly furthers disadvantage. However, the issues are multifaceted, and language of instruction is not sufficient in and of itself to turn around poor academic achievement in resource-constrained poor areas.

There are a number of other clear findings from the LOITASA study. First, the tension between promoting a language of wider communication like English and local languages like isiXhosa is a tension present in many other contexts and countries. The LOITASA Project provides clear evidence of learners' competence in English not suffering as a result of extending the use of local languages as media of instruction. Second, the importance of involving local communities in supporting their children's education is a crucial factor in the project. Such parental and community support is more possible if local languages are used in education. Third, the LOITASA Project emphasises the importance of teacher training in realising the use of local languages as media of instruction. Fourth, the development of terminology in local languages was necessary. Fifth, materials had to be developed in local languages for these languages to be used as academic languages. And finally, negative attitudes toward local languages as media of instruction had to be consciously counteracted. We are aware that the LOITASA Project is not the only one looking at the role of language in learning subjects like mathematics. Phakeng has written extensively about mathematics education and language diversity (see, for example, Phakeng, 2016; Phakeng and Moschkovich, 2013). She consistently argues for a multilingual approach in teaching mathematics, so that learners can draw on their full linguistic repertoires when grappling with mathematical concepts. 


\subsection{Rwanda}

While the current Rwandan policy appears to be taking a giant step away from equity in its promotion of English-medium instruction, recent research offers promising lessons for the improvement of both the quality and equity of classroom practice. In 2008, English became the medium of instruction across all levels of the Rwandan education system (Rosendal, 2011). This was modified in 2011 so that the first three years of primary schooling reverted to learning in Kinyarwanda with English a compulsory subject. While this has many similarities with language-in-education policies across East Africa and farther afield, it is remarkable in its break from the ex-colonial language and the political decision to rebrand Rwanda as an English-speaking nation (Samuelson \& Freedman, 2010). While there has been attention paid to the policy shift in the academic literature (Samuelson \& Freedman, 2010; Assan \& Walker, 2012), much of this has focused on the political and economic rationale and implications. There has been surprisingly little written about its impact on teaching and learning opportunities in classrooms across the country.

The Improving Learner Outcomes through Language Supportive Textbooks and Pedagogy project was designed to contribute to the understanding of this impact and explore ways to support both teachers and learners toward meaningful learning opportunities when children learn in English. The focus was on learners in primary grade 4 , the year when children switch to EMI learning. The project was developed from the understanding that there remain policy imperatives for many low-income countries to promote EMI, and that it is important to identify the challenges and suggest ways to enable inclusive learning in English so that children learning within these policy demands are not disadvantaged. The baseline findings, both in learner language tests and classroom observations, clearly demonstrated that learners do not have the level of English required for epistemological access to the wider curriculum (Milligan et al., 2014). Furthermore, most of the teachers observed were not proficient in the language of instruction.

Learners in these classrooms were given language-supportive textbooks, which were designed to be accessible and to develop their English. Teachers were trained in language-supportive pedagogy. Central to this is the use of the materials and associated activities to support learners to understand new concepts, in say, mathematics. Sanctioned use of Kinyarwanda was encouraged through set spoken activities and glossaries in the textbooks. This was to support learners' access to new topics, as spoken activities were designed to facilitate initial understanding and the glossary provided key vocabulary. Interviewed during the Improving Learner Outcomes through Language Supportive 
Textbooks and Pedagogy project, learners spoke passionately about the difference it made to have materials that allowed the use of Kinyarwanda:

The former books had difficult English and there were no meanings in Kinyarwanda and this made it difficult for us in rural areas to understand. But these books are easy to read and understand because they have new words explained in Kinyarwanda. This helped us increase our knowledge. (Unpublished project metadata)

This statement is particularly interesting as it highlights the potential for language-supportive textbooks to enable greater epistemological access to the wider curriculum for all children.

The study's evaluation suggests that a language-supportive approach had a significant impact both on learner outcomes and their levels of participation in the classroom (Milligan, Clegg, \& Tikly, 2016). More positive test scores were witnessed across the learner groups, including those that had very low English proficiency in the pretests. Classroom observation data showed that all learners regularly engaged in speaking, writing, and reading activities, supported by both their access to their own textbook and the promotion of more activities in each lesson. Pedagogical practice witnessed in classroom observations was very different by the end of the intervention compared to that observed during the baseline study, with significantly more participation.

There are two clear findings from this study. First, it again highlights the way that language is inextricably linked with pedagogic choices in the classroom. As a Kigali teacher cited in the 2014 Education for All Global Monitoring Report eloquently explains:

There remains no doubt that the main barrier to basic education is the forced use of English as medium of instruction. ... [It] not only impedes learning for the children, but is also a major challenge for Rwanda's teachers. Without adequate knowledge in English, teachers are unable to interact with the students, and the result is a strict chalk-and-talk structure. (UNESCO, 2014f, p. 297)

As discussed by Stephanie Bengtsson, Mamusu Kamanda, Joanne Ailwood, and Bilal Barakat in Chapter 10, and Yusuf Sayed and Kate Moriarty in Chapter 9, teacher confidence and capabilities are essential to the achievement of quality education in SDG 4. Insights from teachers from the Rwanda study clearly show the ways that teaching in a language in which they are not proficient 
impacts the pedagogic choices they make, which, in turn, leads to less participatory and inclusive teaching methodologies. This echoes findings from a British Council project, funded under the same Innovation for Education fund, where teachers were supported to improve their English proficiency in recognition of the limitations that their lack of English fluency placed on teaching quality (Simpson, 2013).

Second, the findings show that the debate over language of instruction does not need to conclude with an either-or decision. There are ways that classrooms can be more inclusive even when learning is happening in English, but this rests on rejection of the misguided notion that English-medium classrooms must mean complete immersion in the language of English. To develop the understanding of key concepts, children need to be able to speak, write, and read, and this can be supported by the use of Kinyarwanda, even if the education authorities insist on a policy of English as medium of instruction.

\subsection{Cambodia}

Even if the contexts are not postcolonial, Southeast Asian countries like Cambodia are also multilingual due to the presence of diverse indigenous and immigrant groups. Recently, Cambodia in particular has taken great strides toward improving access and equity in education for indigenous people through implementation of L1-based multilingual education (MLE), providing encouraging lessons for both policy and practice. The dominant language, Khmer, is spoken by the majority of the population and is the constitutionally mandated medium of instruction (Kosonen, 2013). This has long excluded speakers of an estimated 24 indigenous and other nondominant languages from accessing formal education (Simons \& Fennig, 2017; CARE, 2010). In 2002-2003, working in one of the five highland provinces in the northeast where speakers of nondominant languages constitute the numerical majority, CARE International initiated a community-based schools programme of L1-based MLE as part of a larger intervention on behalf of indigenous girls and women. Working closely with provincial and national education officials and with partners such as UNICEF and the NGO International Cooperation Cambodia (ICC), ${ }^{4}$ CARE developed a model whereby community school management committees were organised and empowered to establish their own schools. Rather than merely constructing schools, these committees made a wide range of decisions about how the schools would function, including which community members should be trained as bilingual teachers, recognising that there were few if any 
qualified teachers in the government system with proficiency in indigenous languages.

Beginning in two languages, Tampuen and Kreung, in six schools in the province of Ratanakiri, the programme has expanded over time to additional languages and schools in Ratanakiri and the other four highland provinces. The Ministry of Education, Youth, and Sport (MoEYS) has been integrally involved in the implementation and expansion of MLE, approving a series of legal documents and policy statements institutionalising the approach. In the 15 years since the first MLE community schools opened, five nondominant languages Brao, Bunong, Kavet, Kreung, and Tampuen - are being used for literacy and instruction, and two others - Jarai and Kuy - are in the process of being adopted (Benson \& Wong, 2017). Community teachers have been granted semiprofessional (contract) or professional (state teacher) status based on established criteria, and qualified teachers who speak Indigenous languages have been trained as bilingual teachers, expanding the number of MLE programs serving indigenous learners. Over 4,00o indigenous children were attending MLE classes in 2015 (Nowaczyk, 2015). By 2017, MLE under MoEYS had expanded to over 55 preschools and 80 primary schools throughout the five highland provinces (Benson \& Wong, 2017).

Overwhelmingly positive results have been reported qualitatively in terms of parent and community satisfaction with how students are learning and teachers are teaching (e.g., Benson, 2011). A longitudinal study of learner achievement showed that MLE learners performed better in mathematics than non-M LE learners, and that there were no significant differences between MLE and non-MLE learners in L2 Khmer (Lee, Watt, \& Frawley, 2015). Moreover, in northeastern Cambodia as in other contexts, it is likely that additional advantages accrue because MLE students maintain their home languages, identities, and connection to family and community, all of which are known to build learner self-esteem and facilitate learning (Ball, 2010). Assessments of L1 writing demonstrate clear advantages to MLE learners with regard to literacy skills and self-expression (Benson \& Wong, 2017).

As mentioned above, the Cambodian national government has supported MLE in policy as well as practice, most recently with a 2013 prakas (sub-decree) and a 2015 action plan for education. The Prakas on the Identification of Languages for Khmer [Cambodian] National Learners Who Are Indigenous People (KoC, MoEYS, 2013) frees indigenous learners from the stipulation in the 2007 Education Law that Khmer be the sole language of instruction. This legal opening allows for consistency in implementing the Multilingual Education 
National Action Plan (MENAP), covering the period of 2015 to 2018 (KoC, MoEYS, 2015). This plan describes a series of aims to:

- provide access to quality and relevant education for indigenous learners;

- build capacity of national and subnational education officials to manage and monitor MLE implementation;

- scale up MLE provision in the five designated provinces; and

- promote demand for quality MLE among school management committees, parents, and local authorities.

The plan also spells out a series of implementation steps, including capacity building for trainers and teachers, development of teaching and learning materials, expansion of MLE to new schools and languages, and conversion of all community schools to state schools (Benson \& Wong, 2017). The MENAP along with prior documents effectively gives educational implementers in the five highland provinces the authority to expand MLE to indigenous learners.

Despite these advances, challenges remain. The most urgent may be the act that the original bilingual model adopted and enshrined in policy allows for L1 use only through grade 3, with a transition to Khmer-medium instruction by grade 4 . While the policy also calls for upper primary teachers to be equipped to offer L1 support (Nowaczyk, 2015), the reality is that most trained MLE teachers are needed for placement in grades 1 through 3 . There have been efforts to gain MoEYS approval for piloting a six-year MLE model that would be more theoretically sound and would potentially lead to more robust student outcomes, but thus far authorities are reluctant (Benson and Wong, 2017). Another challenge that has yet to be resolved is how to streamline government approval of orthographies for languages beyond the original five, since the additional two mentioned above have been languishing in the system for years, making it impossible to submit other languages or to move ahead with teaching and learning in those languages.

Other challenges have grown out of the very thing that makes the Cambodian case special, that is, that the government has taken ownership of important aspects of policy and implementation of MLE as developed and practised through a grassroots, community-centred programme. In the process of institutionalising MLE, and as all community schools have been converted to government schools, some of the more community-friendly aspects are falling away. One of those aspects was an alternative school calendar that conformed to the agricultural seasons when learners would be needed by their families, thus improving attendance. MLE expansion has apparently made this unworkable due to national reporting of final grades on one date for all learners in the country. Another is the conversion of the community school management committees, which were created to support MLE, into school support committees (like 
parent-teacher associations) like those non-MLE schools have; it is not clear if this change is in name only or if it involves loss of decision-making power for community members. Another aspect that is currently threatened is the specific teacher training done through each $\mathrm{L} 1$ (Personal communication with CARE staff, June 2017), which is rendered impossible due to the government's requirement of using nationally certified trainers instead of CARE-trained MLE specialists who are indigenous but not certified. Regarding this latter issue, there is reason to be hopeful for a return to effective L1-based training practices, since indigenous teachers are rapidly gaining access to higher levels of training and certification.

\section{5}

\section{Discussion}

Across the three country discussions, there are some clear wider lessons for the implementation of SDG 4. The first is that language of instruction is central to learning. Across the studies, findings show that learners achieve better outcomes when they are learning through MLE or at least through an L1-supported approach. For example, in science and mathematics, all three countries have seen significantly better results when learners' languages are part of instruction. This is consistent with findings in low-income contexts such as Cameroon (Laitin, Ramachandran, \& Walter, 2016), Ethiopia (Heugh et al., 2012), and the Philippines (Walter \& Dekker, 2011), and suggests that the learning of mathematics and science is facilitated by full or partial L1 instruction. Our case studies also offer clear evidence that learners are more engaged and participatory in classrooms when they understand what is going on - a fact which seems blatantly obvious, yet often eludes stakeholders and policymakers due to their histories and ideologies. While a shift to MLE should not be viewed as a panacea, for the millions of children learning in multilingual contexts, there is enough evidence to suggest that implementing MLE provides a significant step forward, not only toward more meaningful learning but also toward equitable education for all.

If we assume that meaningful learning can only take place when there is meaningful teaching, it is clear that language of instruction policy choices also have a significant impact on teacher confidence and pedagogic choices. Equitable access to materials in learners' (and teachers') own languages can also support meaningful learning. Multilingual materials have been shown to be a key resource for children's epistemological access in Rwanda. This is also being increasingly recognised in South Africa, where the Pan South African Language Board, the lexicography units for the official languages, and the 
Department of Basic Education have embarked on a bilingual picture dictionary project for each of the nine official indigenous languages for children up to grade 3. It is hoped that children will use the dictionaries to build on their knowledge, but also acquire vocabulary in English (Langa, 2017). In Cambodia, MLE classes in grades 1 through 3 have access to textbooks, which are based on the national curriculum and relevant storybooks in the five approved indigenous languages. The goal would be to expand in the upper primary grades to Li-based or bilingual content materials, allowing for a more theoretically sound six-year MLE model.

In all three cases, there are clear policy and parental demands for the dominant language. In light of the evidence-based support for Li-based instruction, we would advocate for high-quality dominant language teaching. This requires the availability of teachers who are proficient in the language and who have the necessary material and pedagogical resources. While we have focused on primary education, it cannot be assumed that all children will be fluent in a dominant language before secondary school starts. Desai (2016) has shown how home languages can facilitate learning even in university contexts. Indeed, colleges and universities are where teachers and other educational personnel are trained; they need to offer courses in both or all languages needed for instruction.

Finally, it is clear that policy reform takes time, that parents and community members need to be integrally involved, along with teachers, and that significant collaboration across government, $\mathrm{NGO}$, and community actors is not only desirable but also necessary. The case study from Cambodia has particularly highlighted the effectiveness of multilevel collaboration, and it may serve as a role model for other countries regarding how MLE for speakers of nondominant languages can be effectively implemented in practice as well as in policy. The initial establishment of MLE as a community-based and communitycontrolled form of education has given indigenous learners access to schools and learning in languages they understand, and similarly has given indigenous community members opportunities to be trained as teachers in their own languages. The establishment of pathways for career development has improved conditions for community teachers and has brought qualified teachers who speak indigenous languages into MLE classrooms (Benson \& Wong, 2017). The remarkable collaboration between the development partners and the Cambodian government has resulted in the development of structural support for MLE implementation - something that is difficult to establish with projectbased educational innovations that have beginning and end dates. Based on their multilevel research in communities, classrooms, and provincial and national education offices, Benson and Wong (2017) argue that the successful 
implementation of MLE in Cambodia is a testimony both to the respectful relationship built by CARE and its development partners with MoEY, and to the demonstrated effectiveness of MLE in the target communities. This is indeed a model that should be emulated, while respecting the historical and ideological particularities of each context.

Based on the evidence presented in this chapter, SDG 4 will not be achieved if education systems continue to deny quality education for all learners, which draws on learners' own linguistic resources. Language considerations need to be more central to the planning and delivery of quality education so that all learners and their communities can be included, not marginalised. In this way, language-of-instruction choice must be seen as a central enabler or disabler of learner access to the curriculum. Across all of the cases discussed in this chapter, it is apparent that there are still large groups of learners who are not exposed to the dominant language outside of the classroom, and whose languages need to be recognised and promoted for educational services to be constructed to adequately meet their needs. Language policies need to reflect inclusivity while recognising the distinctly political nature of the discussion. Here we would like to repeat the concluding words of the NEPI Report in South Africa, which captured the caution that needs to be exercised around choice: 'Language policy for education needs ... to be flexible without being so laissez faire as to allow the perpetuation of present discriminatory practices or ill-informed choices of alternatives to them' (South Africa, NECC, 1992, p. 93). In other words, the state would have to play some kind of interventionist role if we wanted present practices to change. Such intervention would need to be backed up by investing resources in the development of local languages, which would allow the intervention to be operationalised.

At a time when millions of children are still being forced to learn through a dominant language that they do not speak or understand well, where MLE is not yet practised, it is understandable that some consider alternative strategies that can improve the quality of learning, such as oral L1 explanations or language-supportive materials. However, the key message from this chapter is that within discussions of educational quality, equity, and inclusion, there needs to be greater recognition of the importance of implementing a fully developed L1-based MLE program, where all learners are taught and assessed in languages that they speak. More educational resources are needed in local languages, and more teachers need relevant language-related as well as pedagogic training. 
Most importantly, more advocacy at the global, national, and local levels is needed about how languages and literacies are learned to bring about policy and curriculum reform. A real commitment from all parties to recognising and using learners' own languages, and teaching the additional languages needed to access future opportunities in their societies, will contribute significantly to providing more equitable education for all.

\section{Notes}

1 See the school's website for more information: http://thornwood.peelschools.org/ Dual/

2 The NEPI (National Education Policy Investigation) reports were commissioned by the National Education Coordinating Committee, an umbrella body of NGOs, to provide alternative policies in 12 educational areas to assist the first post-apartheid government in South Africa.

3 There has been no formal change to the 1997 Language in Education Policy. More recent documents (RSA, DBE, 2010b) merely describe the school and district contexts with regard to home language distribution and language in education at public schools.

4 ICC had been partnering with UNESCO since 1997 on adult literacy programmes and had developed orthographies for five languages, which were approved by the Ministry of Education, Youth and Sport in 2003 for use in formal education (Nowaczyk, 2015). 